\title{
SEROLOGICAL SURVEY OF MEASLES IMMUNITY IN THE CZECH REPUBLIC, 2013
}

\author{
Hana Tomášková1,2, Hana Zelená1, Alena Kloudová1, Ivan Tomášek ${ }^{1}$ \\ ${ }^{1}$ Public Health Institute Ostrava, Ostrava, Czech Republic \\ ${ }^{2}$ Department of Epidemiology and Public Health, Faculty of Medicine, University of Ostrava, Ostrava, Czech Republic
}

\section{SUMMARY}

Objectives: The aim of the serological survey of measles was to obtain information on the prevalence of antibodies against measles and to verify the effectiveness of vaccination in the Czech population in order to protect public health.

Methods: The serological survey was carried out in the Czech Republic in 2013. Antibodies against measles were tested in 3,111 serum samples of participants aged 1-64 years. Serum samples were tested for the presence of immunoglobulin $\mathrm{G}(\mathrm{lg} \mathrm{G})$ antibodies by enzyme immunoassay (EIA). The vaccination status assessment was based on the medical documentation. Seroprevalence differences were evaluated by sex and age using the Pearson's $x^{2}$ test at $5 \%$ significance level.

Results: The overall seroprevalence reached $93.0 \%(2,893 / 3,111)(95 \% \mathrm{Cl} 92.0-93.9)$. No statistically significant difference was found between men and women $(p=0.724)$. A lower seroprevalence was identified in the first age group ( 1 -year old children) $62 \%(62 / 100)$, as the vaccination has not yet been completed in this age group. The second lowest seroprevalence $80.4 \%$ (160/199) was identified in the age group of $35-44$ years. The highest seroprevalence $97.7 \%$ (387/396) (95\% Cl 95.7-99.0) was in the population with naturally-induced immunity (age above 45 years). In the individuals with two doses seroprevalence reached $94.1 \%(2,081 / 2,212)(95 \% \mathrm{Cl} 93.0-95.0)$. The level of IgG antibodies decreased in persons above 7 years of age.

Conclusions: Based on the results of the serological survey carried out in 2013 in the Czech Republic, it has been decided to postpone the second MMR (measles, mumps and rubella) dose to the age of 5-6 years.

Key words: measles, serological survey, seroprevalence, vaccination

Address for correspondence: H. Tomášková, Public Health Institute Ostrava, Partyzánské nám. 7, 70200 Ostrava, Czech Republic. E-mail: hana.tomaskova@zuova.cz

https://doi.org/10.21101/cejph.a5251

\section{INTRODUCTION}

Despite a considerable progress measles remain globally one of the leading causes of death among children (1). In 2012, measles caused 122,000 of deaths in children under 5 years of age, mainly in developing countries (2). Vaccination commenced in the 60 s of the 20th century significantly influenced a change in the epidemiological situation of measles in Europe. In many countries the endemic transmission of infection was successfully interrupted. The incidence rate decreased to a historical low in the year 2007 to less than 10 cases per population of one million (2). Regardless this achievement, since 2010 an increased incidence of measles has been recorded in Europe. In the years 2010 and 2011 over 30,000 new cases (63-68 cases per population of one million) were reported by the European Centre for Disease Prevention and Control (ECDC) (3). The current epidemiological situation is greatly affected by the existence of certain groups of susceptible persons in the population with less than $95 \%$ immunization coverage with two doses of measles vaccine (4).

In the Czech Republic, the incidence of measles decreased after the introduction of the routine measles vaccinations $(2,5)$. The measles vaccination was introduced in 1969. At the beginning it was a one-dose vaccination of children born in 1968 with the use of the Movivac vaccine (Sevapharma Ltd., Prague, Czech
Republic). Later, two supplemental vaccination campaigns were conducted in 1975-1978 and 1979-1981, targeting children born in the period from 1968-1973 and children aged 13-14 years, respectively, who received a second dose of measles vaccine, aiming at a reduction of the pool of children who are susceptible to measles. Since 1982, a two-dose scheme has been introduced (the first dose was applied at 15 months of age and the second dose 6-10 months later after the first dose) (5-7). In the Czech Republic, the following vaccines were mostly used - Mopavac (Sevapharma Ltd., Prague, Czech Republic, since 1987), Trivivac (Sevapharma Ltd., Prague, Czech Republic, since 1995), Priorix (GlaxoSmithKline Biologicals s.a., Rixensart, Belgium, since 2003) and Priorix-Tetra (GlaxoSmithKline, Biologicals s.a., Rixensart, Belgium, since 2007) (6, 7).

The serosurveys of measles immunity of the population have been conducted in the Czech Republic since 1961, when measles was included into the serological surveys repeated on a regular basis. The last serological surveys were conducted in 2001 (8, 9) and 2013.

The aim of the serological survey of measles was to obtain information on the prevalence of antibodies against measles in the Czech population aged 1-64 years in order to protect public health and to verify the effectiveness of vaccination in relation to age and the vaccination status. 


\section{MATERIALS AND METHODS}

\section{Serological Survey}

The seroprevalence of antibodies against measles was determined within the serological survey of measles, mumps, Bordetella pertussis, and viral hepatitis B in the Czech Republic. The sera collection took place in the period from March to April 2013. A total number of 3,111 sera was collected. The numbers of sera by age group varied from 88 to 103 . A representative sample was obtained from 30 randomly selected districts in all 14 regions (77 districts) of the Czech Republic. Sample size was determined on the basis of the number of individuals falling into the specific age groups (1-64 years) and information about the prevalence of antibodies from the previous serological surveys. The final number of samples was determined to 3,200 , i.e. 100 sera in each age group. In total 32 age groups were tested - the age group 1-24 years by individual years and the age group 25-64 by the interval of 5 years. This age groups distribution was chosen because of monitoring the dynamics of antibodies levels. The ratio of men and women was set to $1: 1$, this ratio is based on the ratio of men and women aged 1-64 years in the population (10). The serum samples were collected from patients of 2-4 paediatricians and 2-4 general practitioners in each district. The number of physicians depended on the district population size and they were randomly selected from the database of the General Health Insurance Company of the Czech Republic. Each physician addressed one respondent of specified sex and age group, i.e. the paediatricians of the age group 1-18 years, the practitioners of the age group 19-64 years. Physicians collected a specified blood sample and filled in a questionnaire that included basic demographic information of each respondent (age, sex, date of birth, place of residence), date of sera collection and information on performed vaccination recorded in the medical documentation.

\section{Laboratory Assay}

The serological survey of measles in 2013 was designed in compliance with the requirements of 16 Member States of the
European Union (the Project ESEN 2 - European SeroEpidemiology Network 2) (11). Serum samples were collected and stored at $-70{ }^{\circ} \mathrm{C}$ before being tested. Serological tests were performed at the microbiological laboratory of the Institute of Public Health. Serum samples were tested for the presence of IgG antibodies by enzyme immunoassay (EIA) using the commercial kit Measles IgG (II) EIA of the Denka company, Seiken, Japan. The individual sera were tested manually. The kit contains 4 standards, which values are expressed in the units of EIA ( $\mathrm{U}=\operatorname{arbitrary}$ units $/ \mathrm{ml})$ : $0-400-1,600-3,200$. The standards are not diluted. The results were expressed in the units of EIA. The values higher than 400 were reported as positive, the values in the range from 200 to 400 were reported as borderline, and values less than 200 were reported as negative. Strongly positive samples with EIA $>3,200$ were not further tested. The samples with borderline results were tested repeatedly. If a different result was found in the repeated test (positive or negative), the sample was tested for a third time, and as the final result the prevailing one was recorded.

\section{Statistical Analysis}

Data from the questionnaires were entered into the software EpiData with suitable edit checks and validations. Laboratory IgG results were directly exported from the analyser. Subsequently, the data were merged through an identification number. The prevalence of positive (seroprevalence) and negative sera was expressed in absolute and relative (\%) numbers out of the total number of tested sera by sex and age groups. For the purpose of statistical analysis, the number of age groups was decreased from 32 (Fig. 1) to 10 as indicated in Table 1 . The seroprevalence with $95 \%$ confidence intervals was introduced based on the binomial distribution. The mean value of $\mathrm{IgG}$ antibodies levels was expressed by median (it was censored data). Information about vaccination status according to the number of doses was processed in absolute and relative numbers by sex and age groups. The seroprevalence and the level of IgG antibodies were evaluated using the Pearson's $\chi^{2}$ test and the Mann-Whitney two-sample test or the Kruskal-Wallis test for more than two samples at a significance level of 5\%. The statistical software Stata version 13 was used for analyses.

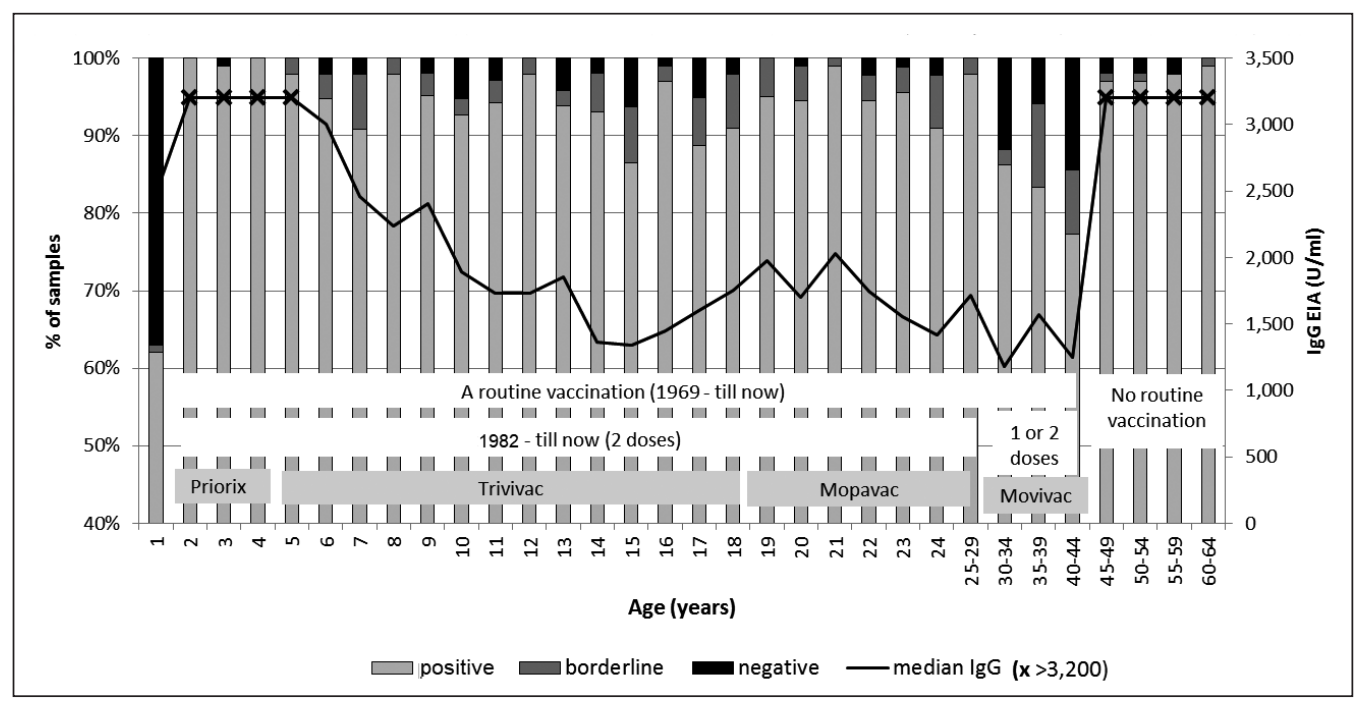

Fig. 1. Proportion of positive, borderline and negative serum samples, median lgG antibodies, information about vaccination and vaccines used in the CR for persons born from 1948-2012. 


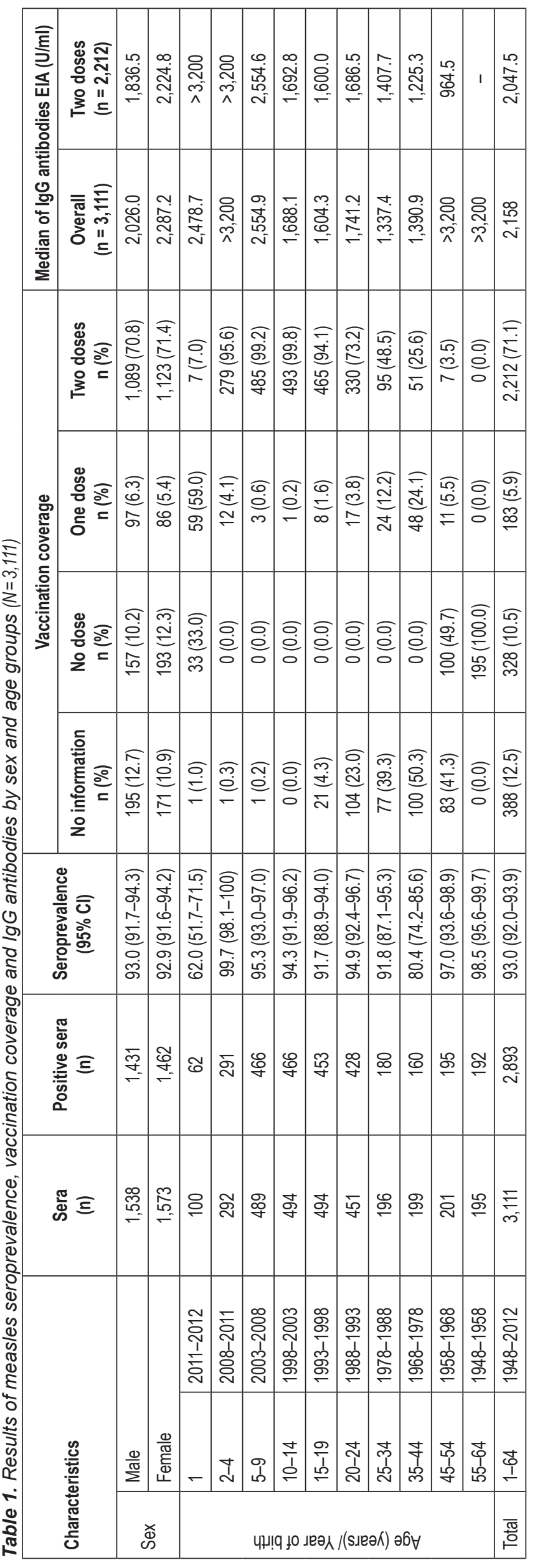

\section{Ethical Considerations}

The study was based on the methodology approved by the Ethical Committee of the Institute of Public Health in Ostrava. Informed consent was obtained from all individual participants included in the study. Each adult respondent signed the informed consent, on behalf of children the informed consent was signed by their parents.

\section{RESULTS}

\section{Sample Description}

Out of the total number of 3,111 persons women accounted for $50.6 \%$ and men for $49.4 \%$. The lowest number of persons was found in the age group of 20-24 years.

\section{Laboratory Results}

In the total study sample $93.0 \%$ positive results, $3.3 \%$ borderline results and $3.7 \%$ negative results were found. No statistically significant difference was confirmed in seropositivity between men and women $\left(\chi^{2}\right.$ test, $\left.p=0.724\right)$ (Table 1$)$. The lowest proportion of positive results was identified in one-year-old children and adults of the age group 35-44 years (Table 1).

The EIA levels of measles IgG antibodies exceeding 3,200 $\mathrm{U} / \mathrm{ml}$ were detected in $32.0 \%(997 / 3,111)$ of the study sample. A detailed description of dynamics of the IgG antibodies levels by specific age groups can be seen in Figure 1. The highest mean levels of IgG antibodies EIA (median > 3,200 (U/ml)) were found in children aged $2-5$ years, then the values decreased up to the age of 14-15 years, and in individuals older than 45 years again the median levels $>3,200(\mathrm{U} / \mathrm{ml})$ were found.

\section{Vaccination}

Except of five cases $(0.03 \%)$ information about vaccination status was collected for all children aged $1-18$ years. In the age group of one-year-old children $33.0 \%$ has not yet been vaccinated and $4.1 \%$ of children from 2 to 4 years were covered with the first dose (Table 1). The proportion of children with two doses was $99.4 \%$ in individuals aged 5 to 18 years.

The situation was much worse in the age groups from 19 to 64 years. The information about vaccination status was unknown in 383 individuals $(28 \%)$ of the total of 1,343 tested subjects. The recorded vaccination coverage under the two doses scheme reached $48.9 \%(550 / 1,125)$ in adults aged 19-44 years.

The analysis by the vaccine used was performed only in children for whom this information was available $(1,728 / 1,768)$. Two doses were applied in 1,652 of children, mostly by the vaccines Trivivac (71.1\%) and Priorix (20.8\%). A single dose received 76 children, the vaccines Priorix (83.9\%), Priorix-Tetra (13.2\%), and Trivivac (3.9\%) were used.

Information about vaccine was known for 510 adults with two doses, the prevailed vaccines were Mopavac (76.1\%) and Movivac (17.5\%). Information about vaccine was available for 86 persons with a single dose and the prevailed vaccines were Movivac (67.4\%) and Mopavac (29.1). 


\section{Relationship between Laboratory Results and Vac- cination Status}

The seropositivity of $94.1 \%$ (95\% CI 93.0-95.0) was identified in persons being vaccinated with two doses, the borderline results were found in $3.7 \%$ and negative results in $2.2 \%$ of persons. Statistically significant differences in the IgG antibodies levels in relation with the number of doses was confirmed in the age groups of 1 year $(p<0.001), 25-34$ years $(p=0.027)$ and $45-54$ years $(p<0.001)$. In the age groups of 1 year and $25-34$ years higher levels of antibodies were found in persons with 2 doses and in the age group 45-54 years the higher levels of antibodies were detected in individuals with naturally-induced immunity (Table 1).

Based on the differences regarding the antibodies levels (Fig. 1) in the age groups 14-18 years and 19-24 years, the analysis of relationship with used vaccines was provided. A significant difference in seropositivity $(91.7 \%,(265 / 289), p=0.032)$ of adolescents (14-18 years) with 2 doses of the vaccine Trivivac was detected compared with individuals (19-24 years) with 2 doses of the vaccine Mopavac $(96.7 \%,(205 / 212))$. This also corresponded with the statistically significant difference $(\mathrm{p}<0.001)$ of $\operatorname{IgG}$ antibodies (median $1,388.0$ vs $1,946.6(\mathrm{U} / \mathrm{ml})$ ).

\section{DISCUSSION AND CONCLUSION}

\section{Laboratory Assay and Evaluation of IgG Antibodies}

Very high level of measles antibodies (EIA values $>3,200$ ) was found in nearly a third of all samples (32\%) tested in 2013. For a more precise determination of the EIA value the test should be repeated by dilution of the sample. Repeated testing of nearly 1,000 samples was not conducted because of very high costs of testing. Determination of the exact values in these samples was not necessary because the very high levels of antibodies have been detected in all cases.

\section{Seroprevalence}

The lowest proportion of seropositivity was found in children under 2 years of age because this group included individuals who have not been vaccinated due to a low age. The second lowest proportion of seropositivity was associated with the age group 35-44 years including individuals who had been vaccinated with a single dose of vaccine according to vaccination schedule. The highest seropositivity was found in individuals with naturallyinduced measles immunity. Comparing with the serological survey 2001 (9), the survey in 2013 achieved similar results. The cohort of 15-34 years with a lower vaccine-induced immunity in 2001 moved to the cohort of 30-44 years of age in 2013 .

\section{Trend in IgG Antibodies Levels}

In 1969, a routine vaccination of children against measles was introduced. Since 1975 a second dose of vaccine has been implemented into the vaccination schedule. The current scheme of vaccination was introduced in 1982, i.e. the first dose of vaccine is applied at 15 months of age and the second dose 6-10 months later after the first dose, i.e. between the 21 st and 25 th month of life. The serological survey from 2013 enabled to evaluate the trend of IgG antibodies levels in vaccinated persons aged 1-31 years (or 1-44 years). As Figure 1 shows there is a rapid decline in the IgG antibodies levels and corresponding lower seroprevalence in children aged 7 years. A significant difference was also found in the seroprevalence level according to the vaccine used. In individuals aged 14-18 years vaccinated with 2 doses of Trivivac a lower median of IgG antibodies was detected contrary to persons vaccinated with 2 doses of Mopavac. Both vaccines were from the same producer (Sevapharma Ltd., Prague, Czech Republic), but Mopavac was a vaccine against 2 infections (measles and mumps) (12) and Trivivac was a triple vaccine (measles, rubella and mumps) (13).

The differences in the levels of IgG antibodies according to the number of doses have only been observed in the age groups that were not vaccinated in 2 doses scheme of the vaccination coverage. In other groups, despite lacking information, the antibodies levels corresponded with vaccination with two doses.

\section{Morbidity}

The effectiveness of vaccination resulted in a relatively small incidence of measles in the Czech Republic ( $<1$ case per 100,000 inhabitants) (2). Only in the year 2014 the incidence has increased to 2.1 cases per 100,000 inhabitants (2). These cases were related mainly to the measles epidemic that occurred in one of the regions in the Czech Republic. Measles most often occurred in persons born in 1970-1980, but were also observed in the youngest, nonvaccinated children (14).

\section{Public Health Implications of Serological Survey 2013}

Based on the results of the serological survey the National Immunization Committee proposed a change in the vaccination schedule; the second dose of measles, mumps and rubella (MMR) was postponed to the age of 5-6 years (15). This recommendation is in compliance with the CDC Vaccine Recommendations (16).

\section{Comparison of Czech Serological Survey 2013 Results with Results from European Countries}

In the member states of European Union, measles vaccination is included into the national immunization programmes which may vary in terms of the specific vaccine doses application. Monitoring of herd immunity through serological surveys is carried out in different countries around the world (17). Based on literature review the measles serological surveys were recently conducted in several countries of the European region, for example in Spain (2011) (18), in the Netherlands (2006-2007) (19), in Italy (2004 and 2005-2006) (20,21), in Belgium (2003) (22) and other countries (23). The results of these studies are consistent with published results. No significant sex differences were found but the studies show different proportion of positive results among age groups depending on vaccination status and natural immunity, and also differences among specific population groups by geographical location of these studies.

In Madrid (Spain), the seroprevalence examined in 1,153 healthy volunteers aged 19-39 years reached 92.1\% (95\% CI: 90.3-93.7) in 2011. No statistically significant differences were found between the sexes (18). 
In the Netherlands, a population-based sero-epidemiological study was conducted in 2006-2007 ( $\mathrm{N}=7,900)$. The overall seroprevalence in the Dutch population was $96 \%$. But there was the problem with the orthodox Protestant minority, the seroprevalence in the orthodox Protestant individuals aged $<10$ years was only $51 \%$ (19).

In Tuscany, Italy, a serological survey was conducted in the years 2004-2006 after the national serological study. Antibodies against measles were tested among 945 persons aged $1-49$ years (20). The seroprevalence in persons aged 1-29 years ranged from 63.2 to $73.9 \%$, in the age group 25-29 years was $84.4 \%$, and among individuals aged 30-49 years ranged from 94.0 to 96.2\%. The total seroprevalence $84.3 \%$ (95\% CI: $81.8-86.5)$ in Tuscany is higher than the total seroprevalence $77.2 \%(95 \%$ CI: 75.7-78.7) in Italy (Italian seroprevalence data from 2004 , $\mathrm{N}=3,093$, age $1-49$ years) (21).

In Belgium, susceptibility to vaccine-preventable diseases was estimated from a serum survey in 2006. Immunoglobulins against measles, mumps, rubella (MMR), and diphtheria at all available ages (1-65 years) were measured by ELISA. Age-standardized overall seronegativity for measles was low (3.9\%). Although a very high population seropositivity has been detected in this study, the World Health Organization's targets for measles elimination were not met in the age group 5-24 years (22).

The seropositivity results in other countries are similar to the results found in the serological survey 2013 carried out in the Czech Republic.

But the prevalence of susceptible persons in the WHO European Region is one of the reasons why the WHO goal - measles elimination cannot be achieved. In the WHO European Region, the goal was to eliminate measles by 2015 (23), but this objective was not achieved (24).

\section{Limitations}

The unfulfilled specified number of 3,200 respondents, obtained $97.2 \%(3,111 / 3,200)$, can affect the reliability of the data and may represent a survey limitation. Most of the missing data appeared in the age group 20-24 years. Young people at this age usually migrate for study or employment reasons from the place of their permanent residence.

Missing information on vaccination in the practitioners' medical records was other problem. Such information was lacking in more than $31 \%(383 / 1,236)$ of persons aged $19-49$ years. This problem is associated with the transition from the paediatrician to a practitioner. The documentation of paediatricians is very well recorded (only $0.03 \%(5 / 1,917)$ of missing data), but the information is not forwarded to practitioners.

The bias due to diagnostics used should be minimal. The quality of commercial kit Measles IgG(II) EIA from Denka, Seiken, Japan, is inspected by the National Reference Laboratory in the INSTAND (25) system, and by means of standardization of the results of tests carried out on 150 sera within the ESEN2 project $(11,26)$.

\section{Acknowledgements}

The project was funded by the Czech Ministry of Health. The analyses were performed with participation of experts from the Regional Public Health Authority of the Moravian Silesian Region.

\section{Conflicts of Interest}

None declared

\section{REFERENCES}

1. World Health Organization. Media centre. Measles [Internet]. Geneva: WHO; 2015 [cited 2016 May 31]. Available from: http://www.who.int/ mediacentre/factsheets/fs286/en.

2. European Centre for Disease Prevention and Control. Surveillance atlas of infectious diseases [Internet]. Stockholm: ECDC; 2016 [cited 2016 May 31]. Available from: http://atlas.ecdc.europa.eu/public/index. aspx? Dataset $=27 \&$ FixDataset $=1$.

3. European Centre for Disease Prevention and Control. Annual epidemiological report 2014: vaccine-preventable diseases. Stockholm: ECDC; 2014.

4. Lexová P. Measles - the importance of the disease and its incidence in Europe. Zpr CEM. 2011;20(3):103-6. (In Czech.)

5. European Centre for Disease Prevention and Control. Vaccine schedule [Internet]. Stockholm: ECDC; 2015 [cited 2016 May 31]. Available from: http://vaccine-schedule.ecdc.europa.eu/Pages/Scheduler.aspx.

6. Limberková R. The history of measles vaccination [Internet]. Prague: The National Institute of Public Health [cited 2016 May 24]. Available from: http://www.szu.cz/uploads/documents/CeM/NRLs/zard_spal_parotitida/ ockovani/historie ockovani.pdf. (In Czech.)

7. Šejda J, Vaništa J, Doutník S. Measles. Prague: Avicenum; 1983. (In Czech.)

8. Kriz B. Multipurpose serological survey - its philosophy and objectives in the Czech Republic. Cent Eur J Public Health. 2003;11 Suppl:S4-6.

9. Mrazova M, Svandova E, Smelhausova M, Sestakova Z, Benes C. The 2001 serological survey in the Czech Republic - measles. Cent Eur J Public Health 2003;11 Suppl:S36-41.

10. Czech Statistical Office. Statistical yearbook of the Czech Republic. Prague: Czech Statistical Office; 2013.

11. Andrews N, Pebody RG, Berbers G, Blondeau C, Crovari P, Davidkin I, et al. The European Sero-Epidemiology Network: standardizing the enzyme immunoassay results for measles, mumps and rubella. Epidemiol Infect. 2000;125(1):127-41.

12. Mopavac [Internet]. [cited 2016 May 24]. Available from: http://www. vakciny.net/pravidelne_ockovani/MOPAVAC\%20PI.pdf.

13. Trivivac [Internet]. [cited 2016 May 24]. Available from: http://www. vakciny.net/pravidelne_ockovani/Trivivac\%20PI.pdf.

14. Trmal J, Limberkova $\mathrm{R}$. Report on a measles epidemic in the Usti nad Labem Region. Epidemiol Mikrobiol Imunol. 2015;64(3):139-45. (In Czech.).

15. Ministry of Health. The protocol of the National Immunization Committee meeting 2015 September 11 [Internet]. Prague: Ministry of Health of the Czech Republic; 2015 [cited 2016 May 24]. Available from: http://www.mzcr.cz/dokumenty/zapis-ze-zasedani-narodni-imunizacnikomisenikokonane-dne-11-92015-na-mz-c_10823_2138_5.html. (In Czech.)

16. Centers for Disease Control and Prevention. Measles vaccination. [Internet]. Atlanta: CDC; 2015 [cited 2016 May 31]. Available from: http:// www.cdc.gov/measles/vaccination.html.

17. Thompson KM, Odahowski CL. Systematic review of measles and rubella serology studies. Risk Anal. 2016;36(7):1459-86.

18. Gonzalez-Escalada A, Garcia-Garcia L, Viguera-Ester P, Marin-Garcia P, Garcia J, Gil de Miguel A, et al. Seroprevalence of antibodies against measles, rubella, mumps, varicella-zoster, and B. Pertussis in young adults of Madrid, Spain. Hum Vaccine Immunother. 2013;9(9):1918-25.

19. Mollema L, Smits GP, Berbers GA, VanDer Klis FR, Van-Binnendijk RS, DeMelker HE, et al. High risk of a large measles outbreak despite 30 years of measles vaccination in the Netherlands. Epidemiol Infect. 2014;142(5):1100-8.

20. Bechini A, Boccalini S, Tiscione E, Pesavento G, Mannelli F, Peruzzi M, et al. Progress towards measles and rubella elimination in Tuscany, Italy: the role of population seroepidemiological profile. Eur J Public Health. 2012;22(1):133-9.

21. Rota MC, Massari M, Gabutti G, Guido M, De Donno A, Ciofi degli Atti ML. Measles serological survey in the Italian population: interpretation of results using mixture model. Vaccine. 2008;26(34):4403-9.

22. Theeten H, Hutse V, Hens N, Yavuz Y, Hoppenbrouwers K, Beutels P, et al. Are we hitting immunity targets? The 2006 age-specific seroprevalence 
of measles, mumps, rubella, diphtheria and tetanus in Belgium. Epidemiol Infect. 2011;139(4):494-504.

23. World Health Organization. Global measles and rubella strategic plan: 2012-2020. Geneva: WHO; 2012.

24. Meeting of the Strategic Advisory Group of Experts on immunization, November 2013 - conclusions and recommendations. Wkly Epidemiol Rec. 2014;89(1):1-20.

25. Mrazová M, Šmelhausová M. Participation of the NRL for rubella, measles, mumps and parvovirus B19 in the EQA system in medical laboratories in Germany. Zpr CEM. 2000;9(9):370. (In Czech.)
26. Tischer A, Andrews N, Kafatos G, Nardone A, Berbers G, Davidkin I, et al. Standardization of measles, mumps and rubella assays to enable comparisons of seroprevalence data across 21 European countries and Australia. Epidemiol Infect. 2007;135(5):787-98.

Received November 16, 2017 Accepted in revised form March 20, 2018 